\title{
SGLT inhibitors and euglycaemic diabetic ketoacidosis: earlier observations rediscovered
}

\author{
SALEM A BESHYAH, ${ }^{1,2}$ BRIAN M FRIER ${ }^{3}$
}

\begin{abstract}
Diabetic ketoacidosis (DKA) is a life-threatening metabolic derangement, defined by the presence of severe hyperglycaemia, ketosis and metabolic acidosis. Recently, DKA was redefined to be present when the latter two abnormalities occur without severe hyperglycaemia. Munro and colleagues in Edinburgh described 'euglycaemic diabetic ketoacidosis' in 1973 and suggested this new terminology. The same year the critical feature of this subclass of DKA - that is, an increased urinary loss of glucose - was identified by Ireland and Thomson in Glasgow. In the subsequent $\mathbf{4 0}$ years (from 1974 to 2014), clinical interest in this condition was limited to a few case reports. The emergence of a new class of antidiabetes medications, the sodium-glucose cotransporter (SGLT) inhibitors, which promote profuse glycosuria, has reawakened interest in euglycaemic DKA, as this is a side effect of these drugs. The earlier perceptive observations of these physicians in Scotland deserve to be recognised for their contribution in identifying and describing euglycaemic DKA and correctly identifying its underlying pathogenesis. Recent international consensus has provided guidance to physicians to aid timely recognition of the condition by testing for ketosis in the appropriate clinical context and to manage it effectively by discontinuing the SGLT inhibitor and provision of insulin, carbohydrate and hydration (the STICH protocol). This may be particularly relevant in view of the recent licensing developments for use of certain members of the SGLT inhibitors in type 1 diabetes.
\end{abstract}

Br J Diabetes 2019;19:37-41

Key words: DKA, euglycaemic diabetic ketoacidosis, SGLT inhibitor, type 1 diabetes, urinary glucose

Department of Medicine, Dubai Medical College, Dubai, United Arab Emirates

2 Honorary Professor of Diabetes, The Queen's Medical Research Institute, University of Edinburgh, Edinburgh, UK

Address for correspondence: $\operatorname{Dr}$ Salem A Beshyah Adjunct Professor of Clinical Research and Metabolic Medicine, PO Box 59472, Abu Dhabi, United Arab Emirates

Tel: 00971505662723

E-mail: Beshyah@yahoo.com

https://doi.org/10.15277/bjd.2019.214
Introduction

Diabetic ketoacidosis (DKA) is a life-threatening metabolic derangement of diabetes. It has long been characterised by the triad of hyperglycaemia, metabolic acidosis and ketosis. More recently, DKA has been redefined in the guidelines of the Joint British Diabetes Societies (JBDS) as follows: (i) ketonaemia $3 \mathrm{mmol} / \mathrm{L}$ and above or significant ketonuria (more than $2+$ on standard urine sticks); (ii) blood glucose over $11 \mathrm{mmol} / \mathrm{L}$ or known diabetes mellitus; (iii) venous bicarbonate ( $\mathrm{HCO} 3$ ) below $15 \mathrm{mmol} / \mathrm{L}$ and/or venous $\mathrm{pH}$ less than 7.3. ${ }^{1}$ Thus, this metabolic derangement can occur without marked elevation of blood glucose. However, this observation is not novel. The development of 'euglycaemic diabetic ketoacidosis' (euDKA) was described by a group of diabetologists in Scotland in the early 1970s, along with a putative interpretation of its likely pathogenesis. These observations have often been overlooked by authors of subsequent papers on DKA. Citations of the original report (including self-citation) barely exceeded 100 in total over the 45 years since it was published. In this brief commentary, credit is paid to the perception of the physicians who first described and named this metabolic derangement and others who helped to elucidate the underlying mechanism, demonstrating how it has been 'rediscovered' as a side effect of a new class of antidiabetes drugs.

\section{'Euglycaemic diabetic ketoacidosis'}

In 1973, Dr John Munro (Figure 1, left) and his colleagues, which included Professor lan Campbell, a former co-editor of this journal, while working in the Diabetic and Dietetic Department of the Royal Infirmary of Edinburgh published a case series of 211 episodes of diabetic metabolic decompensation. ${ }^{2}$ Thirty-seven of these patients had severe ketoacidosis with a plasma bicarbonate of $10 \mathrm{mmol} / \mathrm{L}$ or less but with a blood glucose level of less than $16.6 \mathrm{mmol} / \mathrm{L}$. All of the patients were young and had type 1 diabetes mellitus, only one of whom had not been diagnosed previously. Vomiting was a common feature, and in all of these cases carbohydrate intake had either been reduced or stopped while administration of insulin had been maintained or increased. Treatment comprised typical parenteral fluid and electrolyte replacement and large doses of intravenous insulin and carbohydrate in the form of $10 \%$ dextrose. Alkali was either withheld or administered sparingly, and serial estimations were made of plasma bicarbonate to monitor replacement therapy. The authors coined the term 'euglycaemic diabetic ketoacidosis' (euDKA) to describe this metabolic derangement; this nomenclature had not been used previously in publications on diabetic ketoacidosis (Figure 2, upper). 
Figure 1. The first authors on the paper and correspondence describing and elucidating the mechanism of 'euglycaemic diabetic ketoacidosis' in 1973. Images are credited to the Royal College of Physicians of Edinburgh and the BMJ

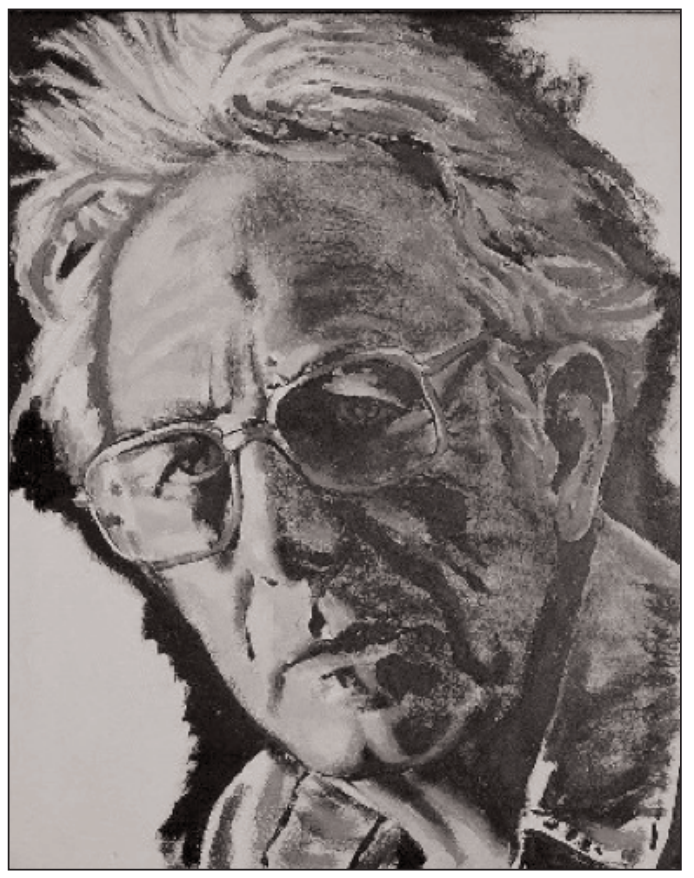

John F Munro (1933-2013)

MBChB, MRCP Edinburgh, FRCP (Edin), OBE

Former Consultant Physician, Eastern General Hospital, Edinburgh and Registrar, Royal College of Physicians of Edinburgh (RCPE). Well known for his work on obesity, medical education and multidisciplinary team working. His support to many countries in Asia and Africa through the RCPE offices is well recognised.

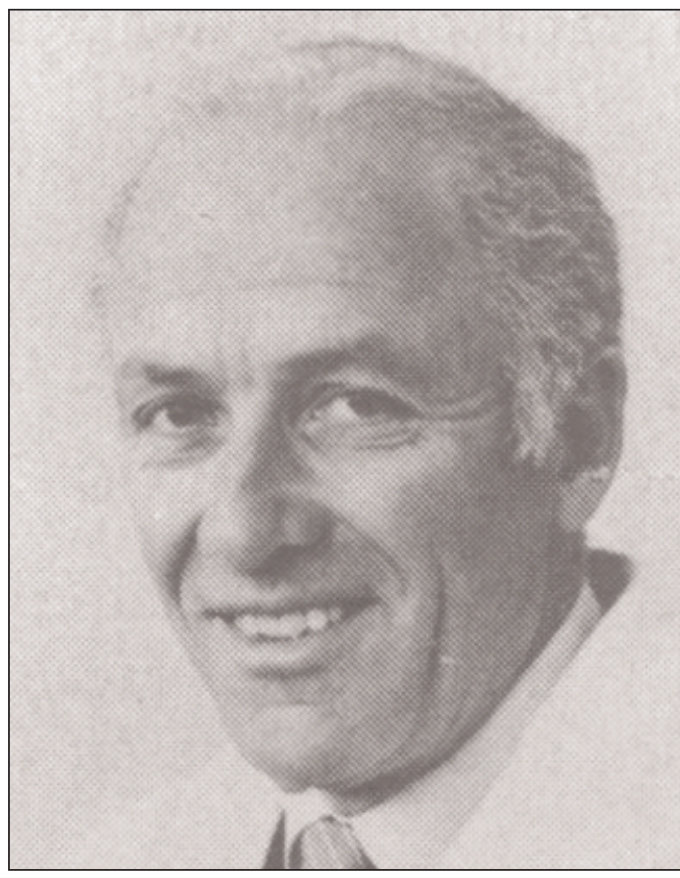

John T Ireland (1932-1987)

MBChB MD, FRCP(Edin), FRCP(Glas)

Former Consultant Physician, Southern General Hospital, Glasgow. He was the founding editor of Diabetic Medicine and co-author of "Diabetes Today" and the "Colour Atlas of Diabetes". His research was on diabetic renal disease and he contributed to the development of technology for the management of diabetes, including the insulin pen.

\section{The concept accepted but the interpretation debated}

Dr John Ireland (Figure 1, right), a consultant diabetologist, and Dr William Thomson, a clinical biochemist in the Southern General Hospital in Glasgow immediately responded to the paper by Munro and colleagues in a letter to the British Medical Journal, ${ }^{3}$ and commended the authors for identifying a subgroup of young patients with insulin-dependent diabetes who had developed ketoacidosis without significant hyperglycaemia (Figure 2, lower). While acknowledging that this observation has extended the range of identifiable metabolic derangements associated with insulintreated diabetes, they commented that, with the exception of vomiting, the clinical features were difficult to explain. They added that in their clinical experience such patients characteristically were young, had good renal function and, in some individuals, a massive urinary excretion of glucose had been observed. These patients appeared to have a lower renal threshold for the excretion of glucose. Since ketoacidosis is generally regarded to be the metabolic consequence of excessive and uncontrolled gluconeogenesis, coupled with increased plasma concentrations of free fatty acids, they observed that it was difficult to postulate that the relative euglycaemia was a consequence of a diminished production of glucose. As an alternative, they proposed that "a greater urinary loss of glucose was a more likely explanation". It was not clear whether this could be a consequence of increased growth hormone secretion affecting renal function, related to the enhanced urinary clearance of glucose that is recognised to be present in early type 1 diabetes, or simply represented one end of the spectrum of the renal threshold for excretion of glucose. ${ }^{3}$

\section{Reawakening of interest in euglycaemic DKA with the introduction of SGLT2 inhibitors}

Subsequent discussion of euDKA and its pathogenesis was sparse. For instance, 88 publications were retrieved from the Scopus database in the last four years (2015-2018) compared with only 30 papers in the previous 41 years (1973-2014). This paucity of publications on euDKA for a protracted period after its original description is depicted in Figure 3. From 1974 to 2014, only a few isolated clinical cases were reported. These were associated with the effects of starvation, excessive alcohol consumption, chronic liver disease and glycogen storage disorders, pregnancy and substance abuse with cocaine. However, the introduction of a new class of antidiabetes medications, the sodium glucose cotransporter (SGLT) inhibitors, ${ }^{4}$ has reawakened interest in euDKA and shed further light on the underlying pathogenetic mechanisms.5,6 The upsurge in 
Figure 2. Images of the original publications in the BMJ showing the titles of the first description by Munro and colleagues (upper panel) suggesting a suitable name for the metabolic derangement and the correspondence by Ireland and Thomson (lower panel) proposing the possible pathogenetic mechanism

\title{
Euglycaemic Diabetic Ketoacidosis
}

\author{
J. F. MUNRO, I. W. CAMPBELL, A. C. MCCUISH, L. J. P. DUNCAN \\ British Medical Journal, 1973, 2, 578-580 \\ blood glucose exceeded $650 \mathrm{mg} / 100 \mathrm{ml}$ or the plasma bicarbonate \\ was less than $15 \mathrm{mEq} / \mathrm{l}$. In 11 there was gross hyperglycaemia \\ Summary \\ Of a series of 211 episodes of diabetic metabolic decom- \\ pensation 37 had severe euglycaemic ketoacidosis (a \\ blood sugar level of less than $300 \mathrm{mg} / 100 \mathrm{ml}$ and a plasma \\ bicarbonate of $10 \mathrm{mEq} / \mathrm{L}$. or less). All were young insulin- \\ dependent diabetics, only one being previously undiag- \\ 1973) This paper considers the other extreme of the broed \\ 1973). This paper considers the ocher extreme of the broad \\ spectrum of diabetic metabolic decompensation-namely, \\ severe ketoacidosis with a plasma bicarbonate of $10 \mathrm{mEq} / \mathrm{l}$. or \\ less without pronounced hyperglycaemia, the blood glucose \\ being less than $300 \mathrm{mg} / 100 \mathrm{ml}$. We have called this syndrome \\ "euglycaemic diabetic ketoacidosis" for lack of a better term.
} nosed. Vomiting was a common factor, and in all carbo-

BRITISH MEDICAL JOURNAL 14 JULY 1973

Euglycaemic Diabetic Ketoacidosis

SIR,-In identifying a group of young diabetics presenting in ketoacidosis without significant hyperglycaemia, Dr. J. F. Munro and hi- collearues (9 June, p. 578) have enhanced the panorama of diabetic metabolic upsets. With the exception of vomiting, however, there was difficulty in explaining the features.

In our experience such patients are characteristically voungsters with good renal function and in some we have identified a massive urinary loss of sugar and a greater tendency to a low renal thre hold to glucose than in others. Since ketoacidosis is generally recarded as the metabolic outcome of excessive gluconeogenesis coupled with increased fatty acid release, it seems difficult to postulare that the relative euglycaemia is due to a lerser glucose formation in such cases. A greater urinary loss of glucose seems to us more likely. Whether this is a consequence of increased growth hormone secretion affecting renal function, related to the ennanced clearance reported in early diabetic renal involvement, ${ }^{1}$ or simply represents one end of the spectrum in terms of the renal threshold to glucose, remains to be determined. Whatever the explanation, perhaps the clue lies in the suggestion by Dr. Munro and his colleagues that there is an ability to grow out of the tendency.-We are, etc.,

Medical Unit,

J. T. IRELAND

Biochemistry Department,

Southern General Hospital,

Glasgow

1 Ditzel, J., and Schwartz, M., Lancet, 1967, 1, 276. interest can be measured by the increasing number of publications from 2015 onwards.

The association between the use of SGLT inhibitors and serious adverse events was highlighted in a Scandinavian register-based cohort study performed between July 2013 and December $2016 .{ }^{6}$ The primary outcomes identified from hospital records were lower limb amputation, bone fracture, diabetic ketoacidosis, acute kidney injury, serious urinary tract infection, venous thromboembolism and acute pancreatitis. In that analysis, the use of SGLT-2 inhibitors, as compared with glucagon-like peptide-1 receptor agonists, was associated with an increased risk of lower limb amputation and DKA, but not with other serious adverse events of current concern. A worrying side effect that has been reported with SGLT-2 inhibitors in patients with type 2 diabetes, and particularly during off-label use in patients with type 1 diabetes, is euDKA. This poses a challenge to physicians as ketoacidosis may be overlooked in sick patients presenting with normoglycaemia, leading to a delay in ap- propriate management. ${ }^{7}$ Although euglycaemia is relatively rare in association with ketoacidosis in people with diabetes, it remains a serious life-threatening medical emergency and must be treated as quickly and effectively as other forms of hyperglycaemic metabolic emergencies. ${ }^{8}$ Consequently, practising physicians should consider the presence of DKA in patients with ketonuria and biochemical evidence of acidosis even when blood glucose levels are not elevated. A high clinical suspicion is required to diagnose euDKA. Blood $\mathrm{pH}$ and blood or urine ketones should be measured in all ill patients with diabetes, irrespective of the presenting blood glucose level. ${ }^{8}$

SGLT inhibitor-associated DKA is likely in patients with insulindeficient diabetes, including those with type 2 diabetes, and is typically precipitated either by the omission of insulin or overzealous reduction in the dose, or with acute illness, dehydration, extensive exercise, surgery, low-carbohydrate diets or excessive alcohol intake. ${ }^{6}$ 
Figure 3. The rarity of euglycaemic diabetic ketoacidosis in the pre-SGLT2 inhibitor era is inferred from the low numbers of papers recorded in Scopus and PubMed between the original description in 1973 and the emergence of SGLT inhibitors for the treatment of diabetes

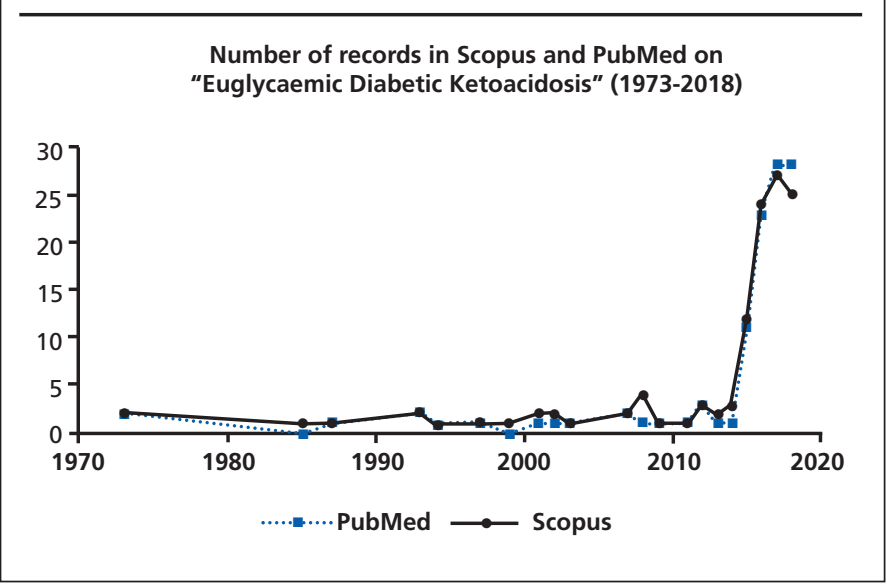

SGLT inhibitor-induced euDKA may occur because of the effects on glucose clearance and volume depletion. Recent data from studies in rodents suggest that a combination of insulinopenia and dehydration are fundamental to the development of euDKA and may be potential therapeutic targets to prevent euDKA occurring in association with SGLT inhibitors. ${ }^{9}$ Patients with types 1 and 2 diabetes who experience nausea, vomiting or malaise while on SGLT inhibitor therapy should have urinary and blood ketones measured as a matter of urgency. $7,8,10$ If urinary ketones are ++ or plasma ketones $>0.6 \mathrm{mmol} / \mathrm{L}$, investigation should proceed to measuring venous or arterial blood gases. A recent international consensus statement provided a good working plan for the risk management of DKA in patients with type 1 diabetes who are being treated with SGLT inhibitors. ${ }^{11,12}$ Prompt verification of the diagnosis, invoking of the 'STICH' (STop the SGLT inhibitor, Inject bolus insulin, Consume $30 \mathrm{~g}$ carbohydrates and Hydrate) protocol, close monitoring and escalation of the care for non-responders should minimise the risk in this context (Table 1).

The licensing status of use of the various SGLT inhibitors in people with type 1 diabetes is controversial (Table 2). The European Commission has approved dapagliflozin for use in type 1 diabetes as an adjunct to insulin in people affected with a BMI of at least 27 $\mathrm{kg} / \mathrm{m}^{2}$, making it the first approval of an oral therapy for type $1 \mathrm{di}-$ abetes in Europe. ${ }^{13}$ The favourable decision was presumably based on data derived from the DEPICT clinical programme in type 1 diabetes. ${ }^{14}$ SGLT-2 inhibitors are currently under regulatory review in the USA and Japan for use as an adjunctive treatment in adults with type 1 diabetes. However, in March 2019 the FDA in the USA rejected sotagliflozin, an oral dual inhibitor of SGLT-1 and SGLT-2. By contrast, sotagliflozin secured a positive opinion from the European Medicines Agency for is ability to lower $\mathrm{HbA}_{1 \mathrm{c}}$ and reduce body weight, despite having an associated DKA rate of 3-4\%. ${ }^{13}$ These licensing changes, while differing on each side of the Atlantic, may potentially place more patients at risk of developing euDKA.
Table 1 'STICH' protocol-based practicalities of management once ketosis has been confirmed in the setting of SGLT inhibitor use in combination with insulin for the treatment of type 1 diabetes

\begin{tabular}{|c|c|c|}
\hline Step & Strategy & Action \\
\hline 1 & Confirm diagnosis & $\begin{array}{l}\text { Verify ketosis by early symptoms } \\
\text { Identify conditions that might be } \\
\text { causing ketosis; }{ }^{*} \text { test for ketones }\end{array}$ \\
\hline 2 & $\begin{array}{l}\text { Invoke the 'STICH' } \\
\text { protocol }\end{array}$ & $\begin{array}{l}\text { STop the SGLT inhibitort } \\
+ \text { Inject bolus insulin } \\
+ \text { Consume } 30 \mathrm{~g} \text { carbohydrates } \\
+ \text { Hydrate }\end{array}$ \\
\hline 3 & Monitor response & Recheck ketones every $3-4$ hours \\
\hline 4 & Escalate care & $\begin{array}{l}\text { Seek emergency medical care if } \\
\text { (a) ketosis does not resolve or } \\
\text { (b) if symptoms of DKA appear, } \\
\text { including abdominal pain, nausea, } \\
\text { vomiting, fatigue, and/or dyspnoea. }\end{array}$ \\
\hline \multicolumn{3}{|c|}{$\begin{array}{l}\text { * Symptoms may start hours after last SGLT inhibitor dose. } \\
\text { † Patients should not take the SGLT inhibitor after ketosis is detected and not take } \\
\text { another dose until ketones have resolved and patient has received appropriate } \\
\text { care. }\end{array}$} \\
\hline \multicolumn{3}{|c|}{$\begin{array}{l}\text { DKA, diabetic ketoacidosis; SGLT, sodium glucose cotransporter; 'STICH', STop the } \\
\text { SGLT inhibitor, Inject bolus insulin, Consume } 30 \mathrm{~g} \text { carbohydrates and Hydrate. }\end{array}$} \\
\hline \multicolumn{3}{|c|}{$\begin{array}{l}\text { A steroid-type wallet card is proposed to facilitate concordant patients' and } \\
\text { doctors' actions. }\end{array}$} \\
\hline
\end{tabular}

The international consensus recommendations are therefore timely and are particularly relevant in view of the recent licensing developments. ${ }^{12}$

\section{Conclusions}

In this short commentary we have highlighted the early Scottish observations on 'euglycaemic DKA' that have helped to identify this condition and to elucidate the potential pathogenetic mechanisms and the risk factors. Postulation of a pivotal role for the increased diuresis as a mechanism of euDKA was advanced several decades before the SGLT inhibitors came into clinical use. The recent development of this drug class for clinical application has provided an opportunity for the pathophysiology of DKA to be revisited and the pioneering work of these physicians to be more widely acknowledged. Knowledge of the risk factors, diagnostic criteria and management of euDKA is essential for clinicians who are required to manage such patients when they present as unselected medical emergencies. The 'STICH' protocol provides a very helpful working plan for clinicians. This is particularly important in view of the recent licensing developments that may potentially put a greater number of patients at risk of developing euDKA.

\section{Conflict of interest: None \\ Funding: Contributions}

Contributions: SAB conceived and wrote the manuscript and BMF revised it extensively. Both authors approved the final version of the manuscript. 
Table 2 SGLT inhibitors currently approved for use in clinical practice

\begin{tabular}{|c|c|c|c|c|}
\hline $\begin{array}{l}\text { Generic name } \\
\text { (Commercial) }\end{array}$ & Manufacturing company & Available strengths & $\begin{array}{l}\text { Regulatory approval } \\
\text { (year) }\end{array}$ & $\begin{array}{l}\text { Relev } \\
\text { (PubN }\end{array}$ \\
\hline $\begin{array}{l}\text { Canagliflozin } \\
\text { (Invokana) }\end{array}$ & $\begin{array}{l}\text { Mitsubishi Tanabe Pharma Corp, } \\
\text { Janssen Pharmaceuticals Inc }\end{array}$ & $100 \mathrm{mg}, 300 \mathrm{mg}$ & $\begin{array}{l}\text { FDA (USA), EMA (Europe) } \\
(2013)\end{array}$ & 865 \\
\hline $\begin{array}{l}\text { Dapagliflozin } \\
\text { (Forxiga) }\end{array}$ & $\begin{array}{l}\text { Bristol-Meyers Squibb Company and } \\
\text { AstraZeneca plc }\end{array}$ & $5 \mathrm{mg}, 10 \mathrm{mg}$ & $\begin{array}{l}\text { FDA (USA), EMA (Europe) } \\
(2014)+\end{array}$ & 877 \\
\hline $\begin{array}{l}\text { Empagliflozin } \\
\text { (Jardiance) }\end{array}$ & $\begin{array}{l}\text { Boehringer Ingelheim GmbH and } \\
\text { Eli Lilly and Company }\end{array}$ & $10 \mathrm{mg}, 25 \mathrm{mg}$ & FDA and EMA (2014) & 945 \\
\hline $\begin{array}{l}\text { Ertugliflozin } \\
\text { (Steglatro) }\end{array}$ & $\begin{array}{l}\text { Merck \& Co Inc } \\
\text { (MSD outside USA) }\end{array}$ & $5 \mathrm{mgs}, 15 \mathrm{mg}$ & $\begin{array}{l}\text { FDA (USA), EMA (Europe) } \\
(2017 ; 2018)\end{array}$ & 58 \\
\hline $\begin{array}{l}\text { Ipragliflozin } \\
\text { (Suglat) }\end{array}$ & $\begin{array}{l}\text { Astellas Pharma Inc and } \\
\text { Kotobuki Pharmaceutical Co Ltd }\end{array}$ & $25 \mathrm{mg}, 50 \mathrm{mg}$ & PMDA, Japan (2014) & 167 \\
\hline $\begin{array}{l}\text { Luseogliflozin } \\
\text { (Lusefi) }\end{array}$ & Taisho Pharmaceutical Holdings Co Ltd & $2.5 \mathrm{mg}, 5 \mathrm{mg}$ & PMDA, Japan (2014) & 83 \\
\hline $\begin{array}{l}\text { Tofogliflozin } \\
\text { (Deberza; Apleway) }\end{array}$ & $\begin{array}{l}\text { Chugai Pharmaceuticals Co, } \\
\text { Kowa Co, and Sanofi S.A }\end{array}$ & $20 \mathrm{mg}$ & PMDA, Japan (2014) & 89 \\
\hline
\end{tabular}

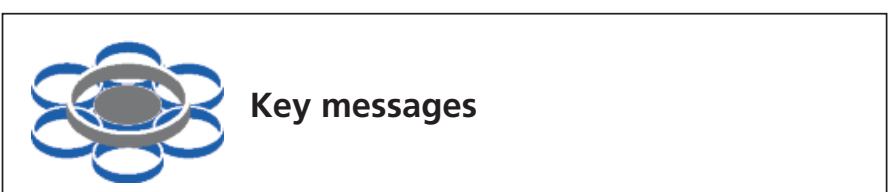

- The development of severe acidosis without hyperglycaemia (euglycaemic ketoacidosis) was described in adult patients with type 1 diabetes in the early 1970s, in which the metabolic derangement was characterised by the presence of profuse glycosuria

- Euglycaemic ketoacidosis has been recognised to be an uncommon but dangerous side-effect of treatment of diabetes with SGLT inhibitors, which promote urinary excretion of glucose, and may be overlooked in ill patients in whom blood glucose is not elevated. The presence of acidosis should be sought

- A useful clinical protocol for the treatment of SGLTassociated euglycaemic ketoacidosis is now available

\section{References}

1. Savage MW, Dhatariya KK, Kilvert A, et al for the Joint British Diabetes Societies. Joint British Diabetes Societies guideline for the management of diabetic ketoacidosis. Diabet Med 2011;28(5):508-15. https://doi.org/ 10.1111/j.1464-5491.2011.03246.x

2. Munro JF, Campbell IW, MacCuish AC, et al. Euglycaemic diabetic ketoacidosis. Br Med J 1973:2(5866):578-80.

3. Ireland JT, Thomson WST. Euglycaemic diabetic ketoacidosis. Br Med J 1973;3(5871):107 (letter).

4. Davies MJ, D'Alessio DA, Fradkin J, et al. Management of hyperglycaemia in type 2 diabetes, 2018. A consensus report by the American Diabetes Association (ADA) and the European Association for the Study of Diabetes (EASD). Diabetologia 2018;61(12):2461-98. https://doi.org/ 10.1007/s00125-018-4729-5
5. Zelniker TA, Wiviott SD, Raz l, et al. SGLT2 inhibitors for primary and secondary prevention of cardiovascular and renal outcomes in type 2 diabetes: a systematic review and meta-analysis of cardiovascular outcome trials. Lancet 2019;393(10166):31-9. https://doi.org/10.1016/S01406736(18)32590-X

6. Ueda P, Svanström H, Melbye, et al. Sodium glucose cotransporter 2 inhibitors and risk of serious adverse events: nationwide register based cohort study. BMJ 2018;363:k4365. https://doi.org/10.1136/bmj.k4365

7. Modi A, Agrawal A, Morgan F. Euglycemic diabetic ketoacidosis: a review. Curr Diabetes Rev 2017;13(3):315-21. https://doi.org/10.2174/ 1573399812666160421121307

8. Rawla P, Vellipuram AR, Bandaru SS, et al. Euglycemic diabetic ketoacidosis: a diagnostic and therapeutic dilemma. Endocrinol Diabetes Metab Case Rep 2017;2017. pii: 17-0081. https://doi.org/10.1530/EDM-170081

9. Perry RJ, Rabin-Court A, Song JD, et al. Dehydration and insulinopenia are necessary and sufficient for euglycemic ketoacidosis in SGLT2 inhibitor-treated rats. Nat Commun 2019;10(1):548. https://doi.org/ 10.1038/s41467-019-08466-w

10. Peters AL, Buschur EO, Buse JB, et al. Euglycemic diabetic ketoacidosis: a potential complication of treatment with sodium-glucose cotransporter 2 inhibition. Diabetes Care 2015;38(9):1687-93. https://doi.org/ 10.2337/dc15-0843

11. Garg SK, Peters AL, Buse JB, Danne T. Strategy for mitigating DKA risk in patients with type 1 diabetes on adjunctive treatment with SGLT inhibitors: a STICH protocol. Diabetes Technol Ther 2018;20(9):571-5. https://doi.org/10.1089/dia.2018.0246

12. Danne T, Garg S, Peters AL, Buse JB, et al. International consensus on risk management of diabetic ketoacidosis in patients with type 1 diabetes treated with sodium-glucose cotransporter (SGLT) inhibitors. Diabetes Care 2019 Feb 6. pii: dc182316. https://doi.org/10.2337/dc18-2316

13. European Medicines Agency. Committee for Medicinal Products for Human Use (CHMP). Summary of opinion (initial authorisation): Zynquista (sotagliflozin). Available at: https://www.ema.europa.eu/en/documents/ smop-initial/chmp-summary-positive-opinion-zynquista_en.pdf (last accessed 1 May 2019).

14. Dandona P, Mathieu C, Phillip M, et al; DEPICT-1 Investigators. Efficacy and safety of dapagliflozin in patients with inadequately controlled type 1 diabetes: the DEPICT-1 52-week study. Diabetes Care 2018; 41(12):2552-9. https://doi.org/10.2337/dc18-1087 